\title{
Writing Field Analysis for Shingled Bit-Patterned Magnetic Recording
}

\author{
X. G. Li, Z. J. Liu, A. G. Kang, and X. Y. Xie \\ College of Physics and Optoelectronics, Taiyuan University of Technology, Taiyuan, China \\ Correspondence should be addressed to X. G. Li; vencent1227@hotmail.com
}

Received 5 April 2017; Revised 7 June 2017; Accepted 18 June 2017; Published 20 July 2017

Academic Editor: K. Devi Chandrasekhar

Copyright ( 2017 X. G. Li et al. This is an open access article distributed under the Creative Commons Attribution License, which permits unrestricted use, distribution, and reproduction in any medium, provided the original work is properly cited.

\begin{abstract}
A novel method utilizing response surface methodology (RSM) is proposed for effective analysis of the combined influence of writing head geometry and media properties on writing field performance. The method comprises two main modules: (1) a parametric writing head model based on finite element electromagnetic field analysis and (2) an effective writing field gradient model based on RSM. Using the method proposed, the writing performance of an asymmetrically shielded writing head for shingled magnetic recording on bit-patterned media (SMR-BPM) is analyzed. The results show that the shielding trailing gap and medium coercivity primarily impact the effective writing field (EWF) gradient and that the shielding side gap has a secondary impact. More importantly, the analysis shows a strong interaction effect between the influences of writing head geometry and medium coercivity on the EWF gradient, which indicates the need for inclusive design.
\end{abstract}

\section{Introduction}

The development of perpendicular magnetic recording is restricted by the famous trilemma caused by the superparamagnetic limit: thermal stability, grain size, and writing ability conflict while the areal density is increasing [1]. In recent years, several new methods and techniques have been proposed to manage this problem, such as heat-assisted magnetic recording (HAMR) [2], microwave-assisted magnetic recording (MAMR) $[3,4]$, shingled magnetic recording (SMR) $[5,6]$, and bit-patterned media recording (BPM) $[7,8]$. In the BPM scheme, the data are stored in patterned magnetic islands instead of grain clusters in conventional continuous media to achieve higher SNR and thermal stability. More recently, it has been demonstrated that the combination of a shingled magnetic recording scheme with bit-patterned media (SMR-BPM) can achieve bit error rates in the range of $10^{-4}$ at an areal density of $10 \mathrm{~Tb} / \mathrm{in}^{2}[9]$ and $10^{-8}$ at $4 \mathrm{~Tb} / \mathrm{in}^{2}$ [10]; $14 \mathrm{~Tb} / \mathrm{in}^{2}$ can be achieved by integrating a heat-assisted scheme [11]. However, for SMR-BPM to fully realize its potential, refined designs for the writing head and recording media are of critical importance. Evaluation of the writing head performance involves precise modeling of relatively complicated geometry features and material properties. And the media characteristics should be considered due to the increasing complexity of the microstructure of the BPM. Research efforts focusing on writing heads [12-15] or BPM [16-18] have been extensive. However, their interdependence and the resulting effects on the writing performance, especially considering the magnetic field distribution around the shingled writing corner, have hardly been addressed, partly due to the complex nature of the problem. In this paper, we use response surface methodology (RSM) [19] to investigate the interaction effect of the head-head and headmedia parameters on the EWF gradient for SMR-BMP. The writing head is asymmetrically shielded, similar to those studied in $[13,15]$. However, it includes a slot between the trailing shield and the side shield on the shingled writing side, as suggested in [20]. The response surface model of the effective writing field gradient is constructed using the central composite design (CCD) [19], and its sensitivity to the shield design parameters and BPM magnetic properties at the critical location of the writing field contour is investigated. The slot has an apparent influence on the writing field angle distribution around the shingled writing corner. Moreover, the response surface analysis shows a clear interaction effect 


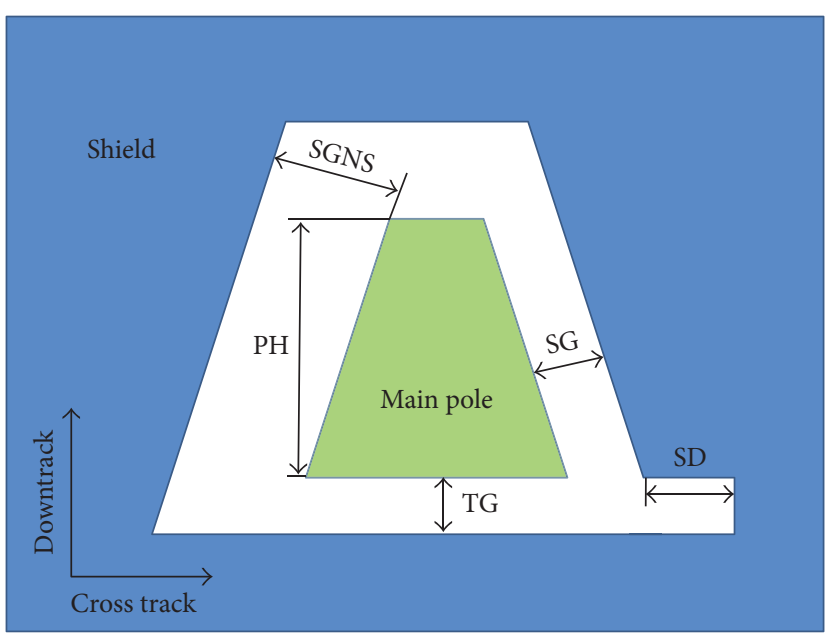

(a)

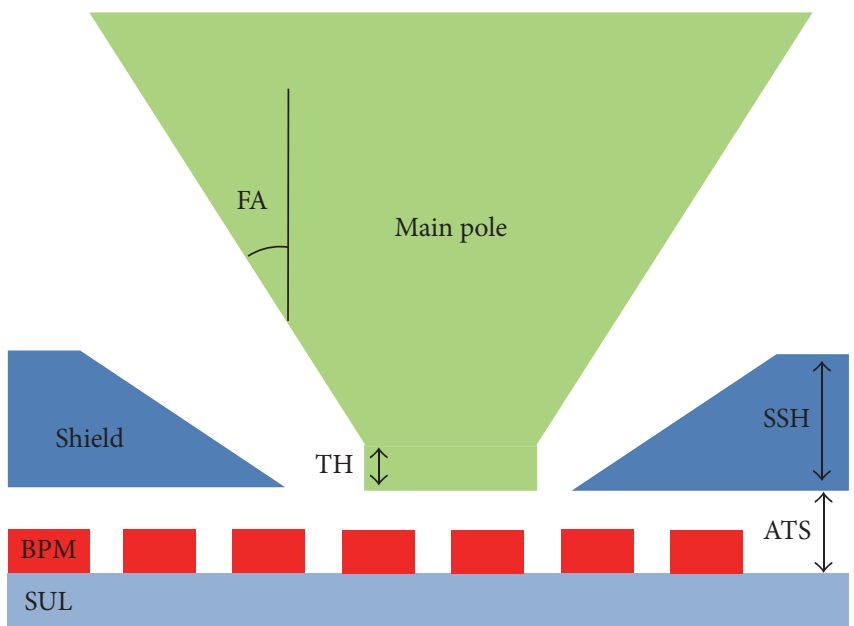

(b)

FIgURE 1: Asymmetrically shielded writing head: (a) top view and (b) downtrack view.

between the shield geometric design parameters and the media properties on the EWF gradient, and such effect may have profound influences on the magnetic switching jitter noise and final bit error rate.

\section{Modeling Details}

2.1. Writing Head Modeling. Utilization of an asymmetrically shielded SMR writing head is believed to help achieve a higher effective writing field and maintain a good field gradient, which is crucial to ultrahigh density recording systems [15]. In this study, an asymmetrically shielded main pole with a slot between the trailing shield and the side shield on the shingled writing side was adopted, and the writing field was analyzed using a finite element Maxwell solver. A schematic view of the investigated writing head structure is shown in Figure 1(a). The shield design parameters considered in the investigation are the trailing gap (TG), side gap on the shingled writing side (SG), and slot depth (SD).

The other parameters were a side gap on the nonshingled writing side (SGNS) of $70 \mathrm{~nm}$, a main pole height $(\mathrm{PH})$ of $75 \mathrm{~nm}$, a throat height $(\mathrm{TH})$ of $5 \mathrm{~nm}$, a main pole flare angle (FA) of $40^{\circ}$, a side shield height $(\mathrm{SSH})$ of $150 \mathrm{~nm}$, and a spacing between the air-bearing surface and the top of the soft-magnetic under layer (ATS) of $25 \mathrm{~nm}$, as shown in Figure 1(b). The saturation magnetization of the main pole was $1910 \mathrm{kA} / \mathrm{m}$, and those of the shield and SUL were $796 \mathrm{kA} / \mathrm{m}$ and $955 \mathrm{kA} / \mathrm{m}$, respectively. Notably, the bitpatterned magnetic islands in Figure 1(b) only represent their relative positions and are not included in the finite element model.

The finite element simulation results show that the length of the slot has a clear influence on the writing field distribution near the shingled writing corner. Figure 2 illustrates the change of writing field angle in the media plane ( $15 \mathrm{~nm}$ below the air-bearing surface) when the slot length is varied from
$10 \mathrm{~nm}$ (Figure 2(a)) to $40 \mathrm{~nm}$ (Figure 2(d)) in an increment of $10 \mathrm{~nm}$. The writing field angle around the shingled writing corner increases with increasing slot length.

2.2. Effective Writing Field and Gradient. A major concern for the recording quality in SMR-BPM is that the writing field pertaining to the writing ability tends to spread around the shingled corner of the writing pole [21]. The EWF gradients at this location, particularly where the effective writing field $H_{\text {eff }}$ exceeds the coercivity of the magnetic island $H_{c}$, are a major factor determining the magnetic switching jitter and the final signal to noise ratio [22]. In this paper, the EWF gradients along the bisector of the main pole base angle (BAB) on the shingle writing side are analyzed using response surface methodology (RSM), as shown in Figure 3.

The magnetization switching behavior of BPM shows clear dependence on the writing field angle, and for magnetic islands with a single phase, the effective writing field can be described by the Stoner-Wohlfarth model [23] as follows:

$$
H_{\text {eff }}=H \cdot\left[(\sin \theta)^{2 / 3}+(\cos \theta)^{2 / 3}\right]^{3 / 2},
$$

where $H$ is the writing field obtained by the finite element solver mentioned above and $\theta$ is the angle between the writing field and the easy axis of the magnetic island, as shown in Figure 2. Notably, exchange coupled composite media with distinct switching field angle dependence [24], which leads to different effective writing field distributions, can also be used to construct the RSM model.

To analyze the influence of design parameters on the writing performance, numerical simulations were devised based on the central composite design (CCD) to construct the second-order response surface model with the EWF gradient as the response target. The model can be expressed as

$$
y=\beta_{0}+\sum_{i=1}^{k} \beta_{i} x_{i}+\sum_{i=1}^{k} \beta_{i i} x_{i}^{2}+\sum_{i<j} \beta_{i j} x_{i} x_{j}+\varepsilon,
$$




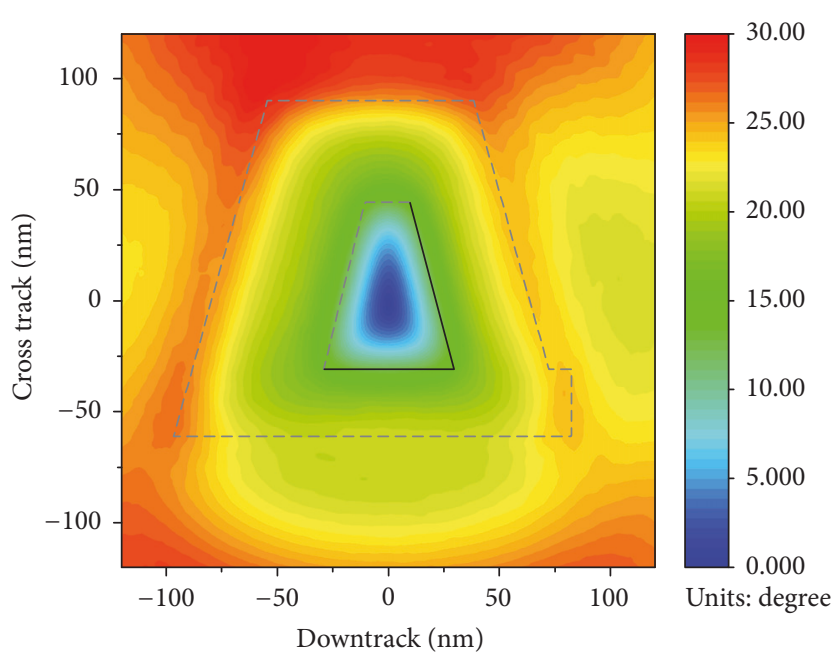

(a)

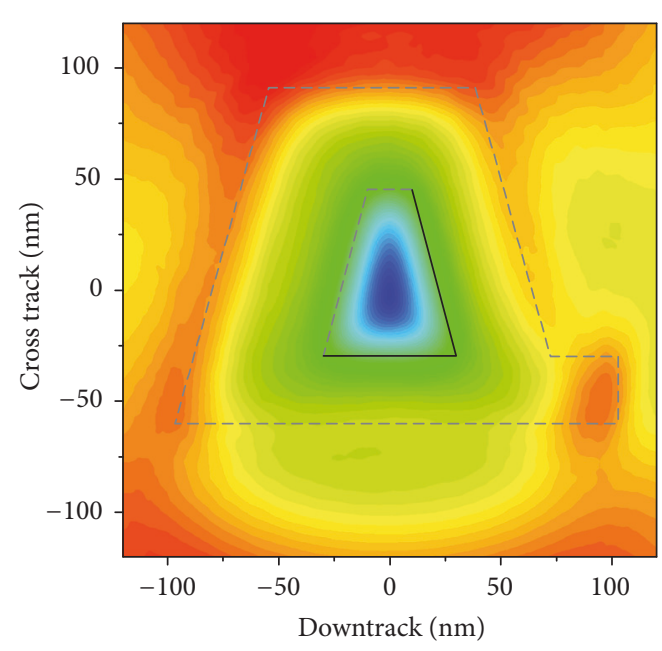

(c)

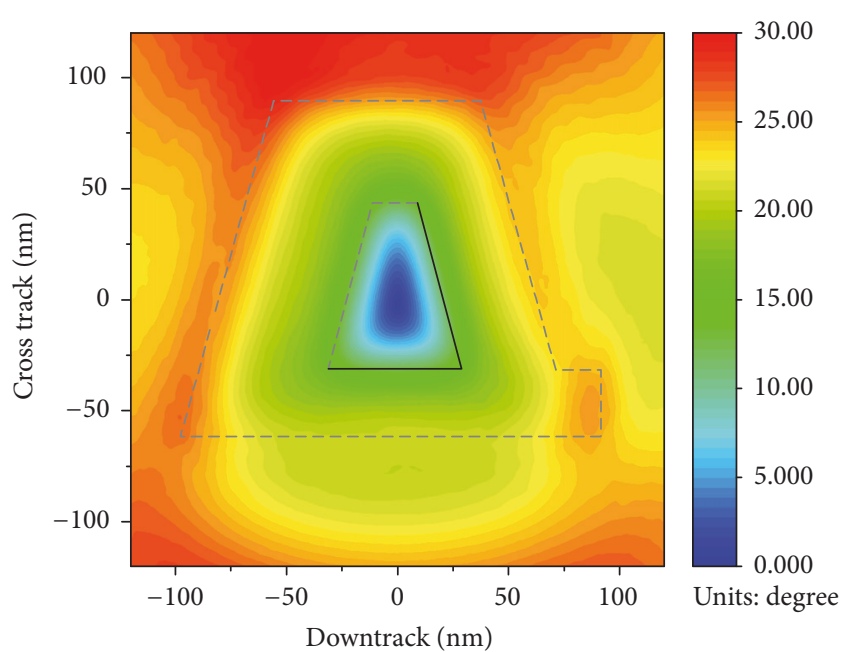

(b)

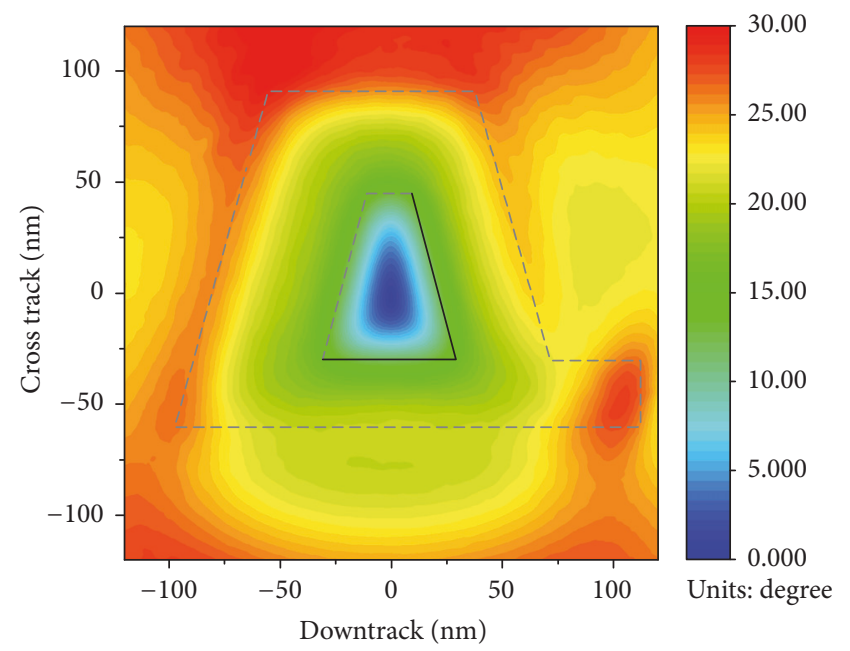

(d)

FIGURE 2: Writing field angle distribution in the media plane with SD varying from $10 \mathrm{~nm}$ (a) to $40 \mathrm{~nm}$ (d). The black solid line indicates the shingled writing corner of the main pole.

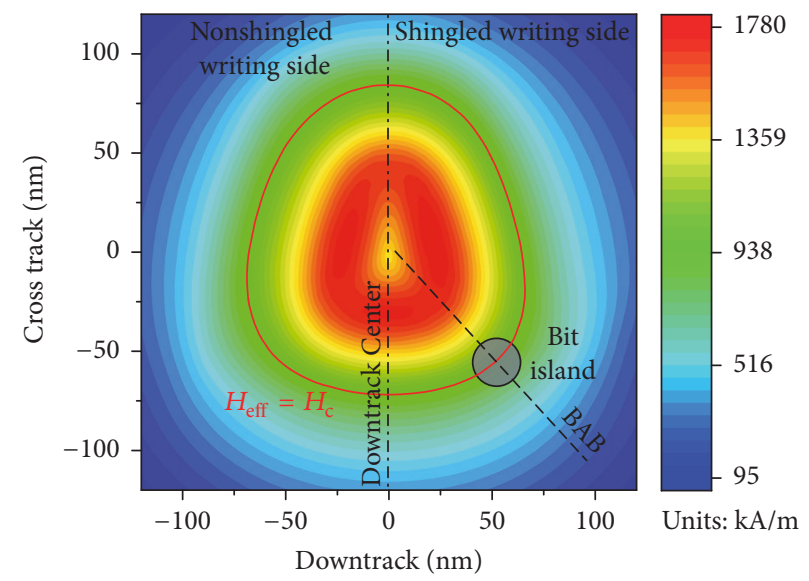

Figure 3: Effective writing field distribution in the media plane. The red solid line represents the field contour $H_{\text {eff }}=H_{c}$. The dashed black line is the bisector of the main pole base angle. 
where $y$ is the EWF gradient obtained by (1), $x_{i}$ is the design parameter, $\varepsilon$ is the fitting error, $k$ is the number of parameters under investigation, and $\beta_{0}, \beta_{i}, \beta_{i i}$, and $\beta_{i j}$ are the coefficients that need to be solved. A typical CCD includes $2^{k}$ factorial runs, $2 k$ axial runs, and $n_{c}$ center runs to identify all the coefficients in the polynomial. In this study, $n_{c}=1$ because the pure error can be eliminated in the numerical simulation approach. Equation (2) can be expressed in matrix form as $\mathbf{y}=\mathbf{X} \boldsymbol{\beta}+\boldsymbol{\varepsilon}$, where

$$
\mathbf{y}=\left[\begin{array}{c}
y_{1} \\
y_{2} \\
\vdots \\
y_{n}
\end{array}\right] \text {, }
$$

$\mathbf{X}$

$$
=\left[\begin{array}{cccc}
1 & x_{11} \cdots x_{1 k} & x_{11}^{2} \cdots x_{1 k}^{2} & x_{11} x_{12} \cdots x_{1 \cdot(k-1)} x_{1 k} \\
1 & x_{21} \cdots x_{2 k} & x_{22}^{2} \cdots x_{2 k}^{2} & x_{21} x_{22} \cdots x_{2 \cdot(k-1)} x_{2 k} \\
\vdots & \vdots & \vdots & \vdots \\
1 & x_{n 1} \cdots x_{n k} & x_{n 1}^{2} \cdots x_{n k}^{2} & x_{n 1} x_{n 2} \cdots x_{n \cdot(k-1)} x_{n k}
\end{array}\right],
$$

$$
\boldsymbol{\beta}=\left[\begin{array}{c}
\beta_{0} \\
\beta_{1} \\
\vdots \\
\beta_{k} \\
\beta_{11} \\
\vdots \\
\beta_{k k} \\
\beta_{12} \\
\vdots \\
\beta_{(k-1) k}
\end{array}\right],
$$

$$
\boldsymbol{\varepsilon}=\left[\begin{array}{c}
\varepsilon_{1} \\
\varepsilon_{2} \\
\vdots \\
\varepsilon_{n}
\end{array}\right]
$$

where $n$ is the total simulation runs. The least square estimation of $\beta$ is

$$
\boldsymbol{\beta}=\left(\mathrm{X}^{\prime} \mathbf{X}\right)^{-1} \mathbf{X}^{\prime} \mathbf{y}
$$

The full RSM model can be further reduced by dropping insignificant terms, which are identified based on an analysis of variance (ANOVA), as discussed in Section 3.

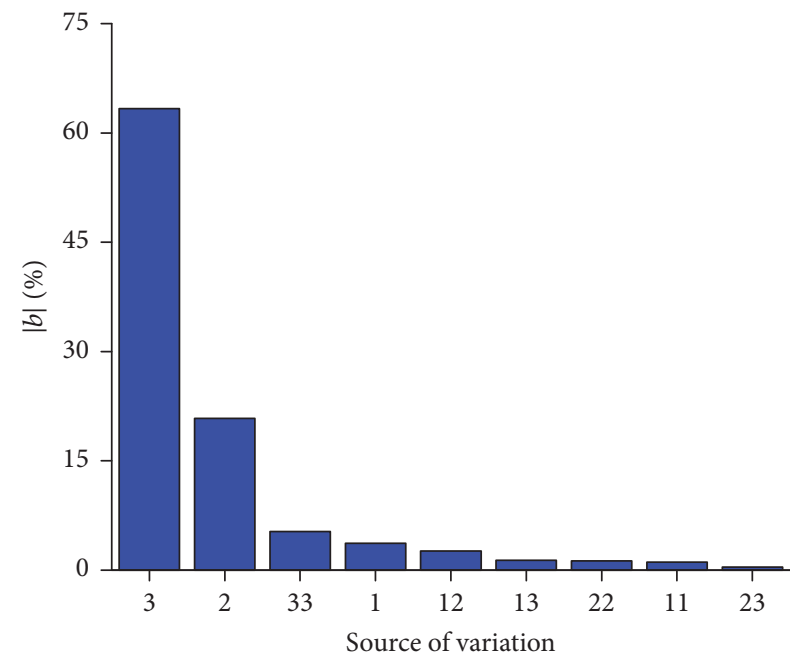

Figure 4: Percentagewise absolute value of coefficients indicating main, interaction, and quadratic effects in simulation design I. As shown on the horizontal axis, 1 indicates the main effect of $X_{1}, 11$ indicates the quadratic effect of $X_{1}$, and 12 indicates the interaction effect of $X_{1}$ and $X_{2}$.

\section{Results and Discussion}

3.1. Simulation Design I. We constructed the EWF gradient response surface models related to SD, SG, and TG to investigate the influence of different head design parameters. The coded and natural levels and the EWF gradient responses are given in Table 1 . The bit-patterned magnetic island with anisotropy energy density $K_{u}=5.83 \times 10^{5} \mathrm{~J} / \mathrm{m}^{3}$ and saturation magnetization $M_{s}=1 \times 10^{6} \mathrm{~A} / \mathrm{m}$ was used for angle dependence calculation.

The main effect and two-interaction effect of the design parameters can be evaluated based on the responses of factorial runs (1-8), and the quadratic effect can be evaluated based on the axial and center runs (9-15). Strength of each effect can be indicated by the percentagewise absolute value of the coefficient in RSM model, as shown in Figure 4. The EWF gradient is dominated by the main effect from $X_{3}$, followed by the main effect from $X_{2}$. A minor quadratic effect from $X_{3}$ can also be observed, and the remaining effects are almost negligible. Figure 5 shows the corresponding half-normal probability plot [25]. The significant effects are far from the reference line and are labeled on the plot.

The second-order response surface model was fitted by least square estimation. Table 2 shows the corresponding analysis of variance. We used the coefficient of determination to evaluate the fitting quality. For the full model, the adjusted coefficient of determination $R_{\text {adj }}^{2}=0.955$, indicating that the model explains $95.5 \%$ of the simulated variation, and the prediction coefficient of determination $R_{\text {pre }}{ }^{2}=0.878$, indicating that the model is expected to explain $87.8 \%$ of the variability in new simulations. Notably, because the pure error can be neglected in the simulation approach, the residual is only due to lack of fit.

The individual significance of each term was investigated using a partial F-test, as shown in Table 2. Because we selected 
TABLE 1: Design details for simulation design I.

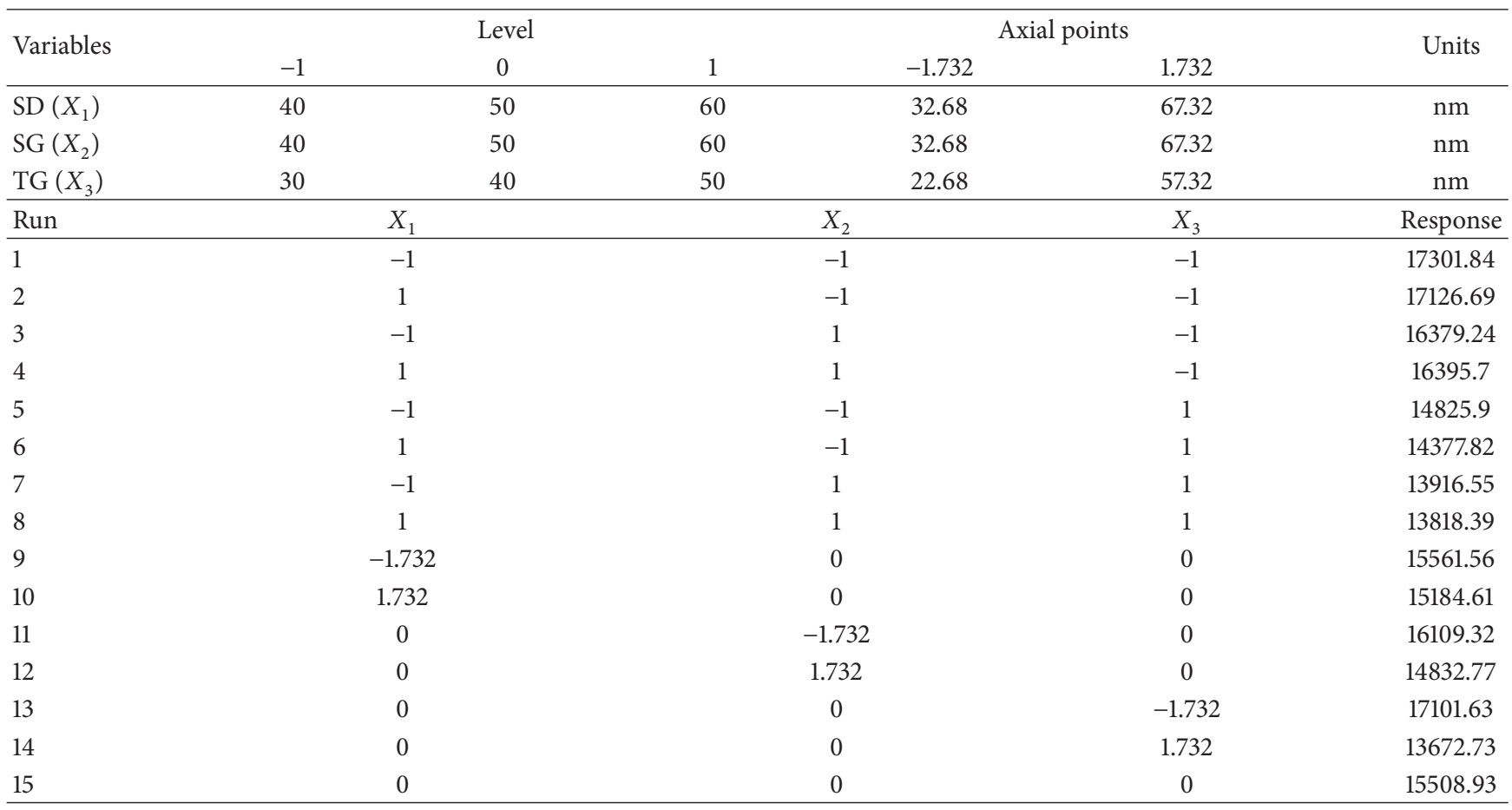

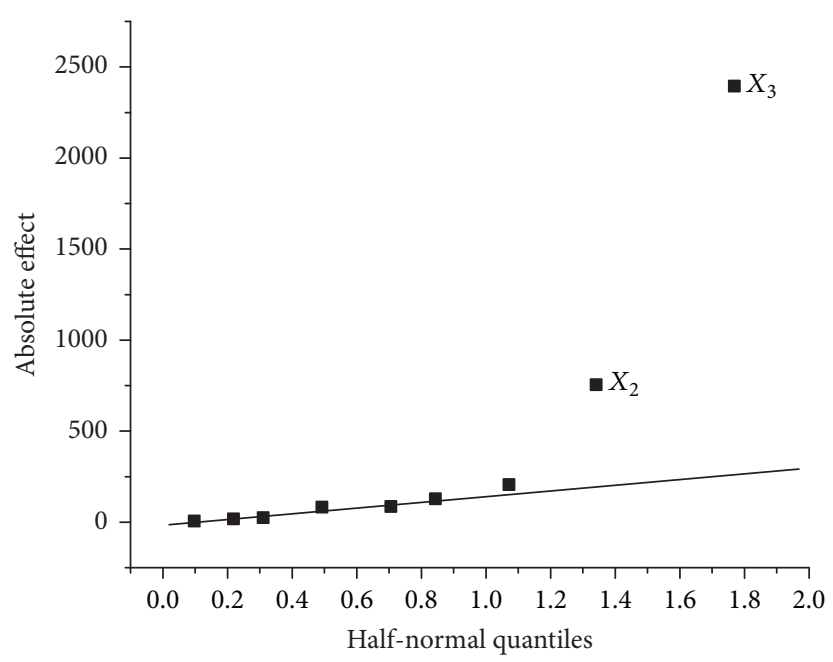

FIGURE 5: The half-normal probability plot for simulation design I.

a significance level of $\alpha=0.05$, only the first-order terms of $X_{2}(P=0.0028)$ and $X_{3}(P=0.000015)$ were identified as significant in the model; thus, the reduced model can be expressed as

$$
\text { Gradient }=\beta_{0}+\beta_{2} X_{2}+\beta_{3} X_{3} \text {, }
$$

where $\beta_{0}=15474, \beta_{2}=-381$, and $\beta_{3}=-1157$, with coefficients of determination $R_{\mathrm{adj}}{ }^{2}=0.97$ and $R_{\mathrm{pre}}{ }^{2}=0.802$. The absence of second-order terms and interaction terms indicates a nearly linear influence of these head design parameters on the EWF gradients. Moreover, the negative coefficients for
$X_{2}$ and $X_{3}$ indicate that a reduction in either SG or TG will improve the field gradient. The EWF gradient is mainly affected by changes in TG, and a relatively small contribution from SG can be observed. Within the design region, optimal design leads to a $20 \%$ increase in the EWF gradient, and the maximum of $17012 \mathrm{~A} / \mathrm{m} / \mathrm{nm}$ can be reached at $X_{2}=$ -1 and $X_{3}=-1$, corresponding to $\mathrm{SG}=40 \mathrm{~nm}$ and $\mathrm{TG}=$ $30 \mathrm{~nm}$. However, SD shows almost no influence under the current simulation settings. A possible reason may be that the magnetic island switching field is not affected by SD because it only modulates the field angle distribution underneath. 
TABLE 2: ANOVA for simulation design I.

\begin{tabular}{|c|c|c|c|c|c|}
\hline Source of variation & Sum of squares & $\mathrm{DF}^{\mathrm{a}}$ & Mean square & $F_{0}$ & $P$ value \\
\hline Regression & 20989736.79 & 9 & 2332192.98 & 34.25 & 0.00058 \\
\hline$X_{1}$ & 131689.24 & 1 & 131689.24 & 1.93 & 0.223 \\
\hline$X_{2}$ & 2031748.24 & 1 & 2031748.24 & 29.83 & $0.0028^{*}$ \\
\hline$X_{3}$ & 18754095.49 & 1 & 18754095.49 & 275.38 & $0.000015^{*}$ \\
\hline$X_{1}^{2}$ & 3952.64 & 1 & 3952.64 & 0.058 & 0.819 \\
\hline$X_{2}^{2}$ & 391.14 & 1 & 391.14 & 0.006 & 0.943 \\
\hline$X_{3}^{2}$ & 2598.20 & 1 & 2598.20 & 0.038 & 0.853 \\
\hline$X_{1} \cdot X_{2}$ & 36655.99 & 1 & 36655.99 & 0.54 & 0.496 \\
\hline$X_{1} \cdot X_{3}$ & 18772.56 & 1 & 18772.56 & 0.28 & 0.622 \\
\hline$X_{2} \cdot X_{3}$ & 4269.09 & 1 & 4269.09 & 0.06 & 0.812 \\
\hline Residual & 340512.37 & 5 & 68102.47 & & \\
\hline Total & 21330249.16 & 14 & & & \\
\hline
\end{tabular}

$R^{2}=0.984 ; R_{\mathrm{adj}}{ }^{2}=0.955 ; R_{\mathrm{pre}}{ }^{2}=0.878 ; \alpha=0.05 .{ }^{\mathrm{a}}$ Degrees of freedom. ${ }^{*}$ Significant terms, $P$ value $<\alpha$.

TABLE 3: Design details for simulation design II.

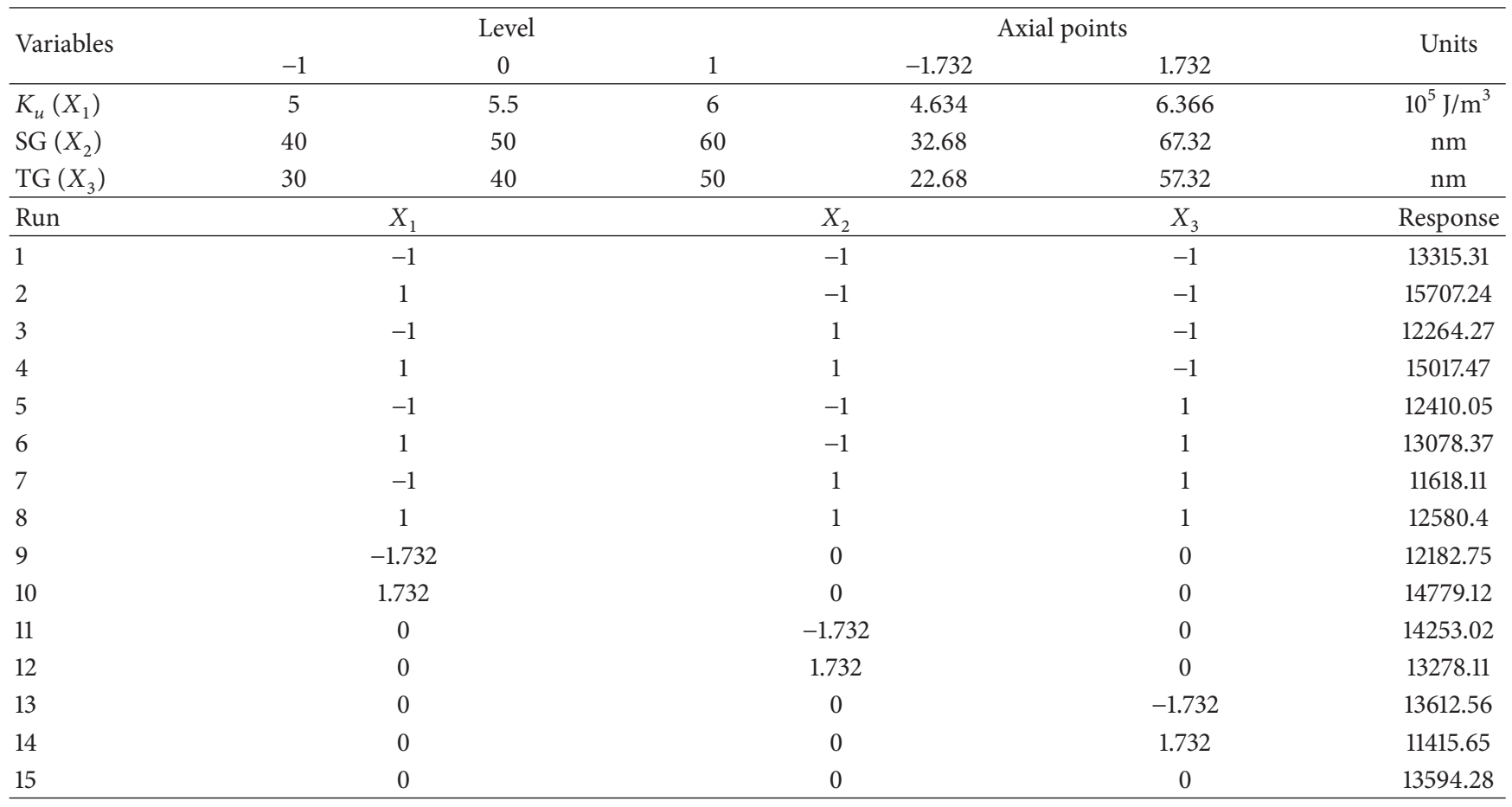

3.2. Simulation Design II. To investigate the relationship between the writing head geometry and bit-patterned media property, another EWF gradient response surface model related to $K_{u}$, SG, and TG was constructed, and SD was fixed at $50 \mathrm{~nm}$. If we assume that the diameter of the magnetic island is $10 \mathrm{~nm}$ and the height is $12 \mathrm{~nm}, K_{u}>3.1 \times 10^{5} \mathrm{~J} / \mathrm{m}^{3}$ gives $K_{u} V / k_{b} T>70$, which satisfies the thermal stability requirement at room temperature. The simulation design and results are shown in Table 3.

Figure 6 shows the effect strength in simulation design II. Variation of the EWF gradient is a relatively complex process in which the main, interaction, and quadratic effects all contribute considerably.
The ANOVA for simulation design II is shown in Table 4. The significant terms are the first-order terms $X_{1}(P=$ $0.000021), X_{2}(P=0.0014)$, and $X_{3}(P=0.000032)$, secondorder term $X_{3}{ }^{2}(P=0.0056)$, and interaction term $X_{1} \cdot X_{3}$ $(P=0.0015)$. Thus, the reduced model is

$$
\begin{aligned}
\text { Gradient }= & \beta_{0}+\beta_{1} X_{1}+\beta_{3} X_{2}+\beta_{3} X_{3}+\beta_{33} X_{3}{ }^{2} \\
& +\beta_{13} X_{1} X_{3},
\end{aligned}
$$

where $\beta_{0}=13617, \beta_{1}=805, \beta_{2}=-337, \beta_{3}=-744, \beta_{33}$ $=-367$, and $\beta_{13}=-439$, with $R_{\mathrm{adj}}{ }^{2}=0.975$ and $R_{\text {pre }}{ }^{2}=$ 0.876 . The positive $X_{1}$ coefficient indicates that the increase in $K_{u}$ improves the EWF gradient. The negative $X_{2}$ and 


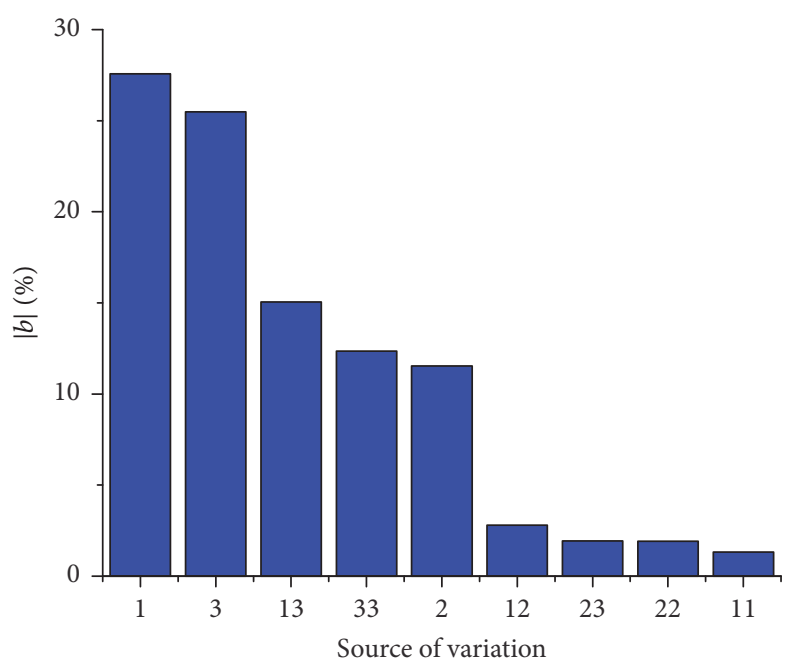

FIGURE 6: Percentagewise absolute value of coefficients indicating main, interaction, and quadratic effects in simulation design II. As shown on the horizontal axis, 1 indicates the main effect of $X_{1}, 11$ indicates the quadratic effect of $X_{1}$, and 12 indicates the interaction effect of $X_{1}$ and $X_{2}$.

TABLE 4: ANOVA for simulation design II.

\begin{tabular}{|c|c|c|c|c|c|}
\hline Source of variation & Sum of squares & DF & Mean square & $F_{0}$ & $P$ value \\
\hline Regression & 21881332.28 & 9 & 2431259.14 & 62.867 & 0.00013 \\
\hline$X_{1}$ & 9076848.13 & 1 & 9076848.13 & 234.709 & $0.000021^{*}$ \\
\hline$X_{2}$ & 1590855.66 & 1 & 1590855.66 & 41.136 & $0.0014^{*}$ \\
\hline$X_{3}$ & 7759224.55 & 1 & 7759224.55 & 200.638 & $0.000032^{*}$ \\
\hline$X_{1}^{2}$ & 9577.97 & 1 & 9577.97 & 0.248 & 0.64 \\
\hline$X_{2}^{2}$ & 20244.24 & 1 & 20244.24 & 0.523 & 0.502 \\
\hline$X_{3}^{2}$ & 834669.40 & 1 & 834669.40 & 21.583 & $0.0056^{*}$ \\
\hline$X_{1} \cdot X_{2}$ & 53667.39 & 1 & 53667.39 & 1.388 & 0.292 \\
\hline$X_{1} \cdot X_{3}$ & 1543974.18 & 1 & 1543974.18 & 39.924 & $0.0015^{*}$ \\
\hline$X_{2} \cdot X_{3}$ & 25413.23 & 1 & 25413.23 & 0.657 & 0.454 \\
\hline Residual & 193364.26 & 5 & 38672.85251 & & \\
\hline Total & 22074696.54 & 14 & & & \\
\hline
\end{tabular}

$R^{2}=0.991 ; R_{\mathrm{adj}}{ }^{2}=0.975 ; R_{\mathrm{pre}}{ }^{2}=0.933 ; \alpha=0.05 .{ }^{*}$ Significant terms, $P$ value $<\alpha$.

$X_{3}$ coefficients are in accordance with (5), and a smaller absolute value of the $X_{3}$ coefficient indicates that its main effect decreases. More importantly, compared with the model in simulation design I, the model is complicated by two extra terms: (1) the second-order term $X_{3}{ }^{2}$, which implies that there is a response stationary point for TG within the design region, and (2) the interaction term for $X_{1}$ and $X_{3}$, which indicates that the influences from $K_{u}$ and TG on the EWF gradient are highly correlated.

Figure 7 shows the response surface of the EWF gradient related to SG and TG when $K_{u}$ was fixed at different levels. The curvature of the surface increases as $K_{u}$ decreases from $6 \times 10^{5} \mathrm{~J} / \mathrm{m}^{3}$ (Figure $7(\mathrm{a})$ ) to $5 \times 10^{5} \mathrm{~J} \mathrm{~m}^{3}$ (Figure $7(\mathrm{c})$ ). Moreover, the response maximum with $K_{u}=5 \times 10^{5} \mathrm{~J} / \mathrm{m}^{3}$ is reached within the design region at $\mathrm{SG}=40 \mathrm{~nm}$ and $\mathrm{TG}=$ $35.2 \mathrm{~nm}$, as shown in Figure 7(c), and a further decrease in TG deteriorates the EWF gradient. This indicates that the optimal TG varies with medium coercivity. Specifically, a medium with lower $K_{u}$ prefers a wider TG. One possible reason is the spatial dependence of the writing field gradient. For a writing head design such as that proposed in [13], the optimal field gradient is confined to a small area in the media plane; thus, the medium coercivity must be carefully designed to achieve improved writing performance.

\section{Conclusion and Perspectives}

In this paper, an EWF gradient model for shingled bitpatterned recording was constructed based on response surface methodology, and the influences from individual and combined parameter variation from the writing head and media were analyzed. Reduction in the trailing gap is more effective than that in the side gap to improve the EWF gradient. The slot between the trailing shield and side shield on the shingled writing side can be used to modulate the field angle distribution but does not show a clear effect on 


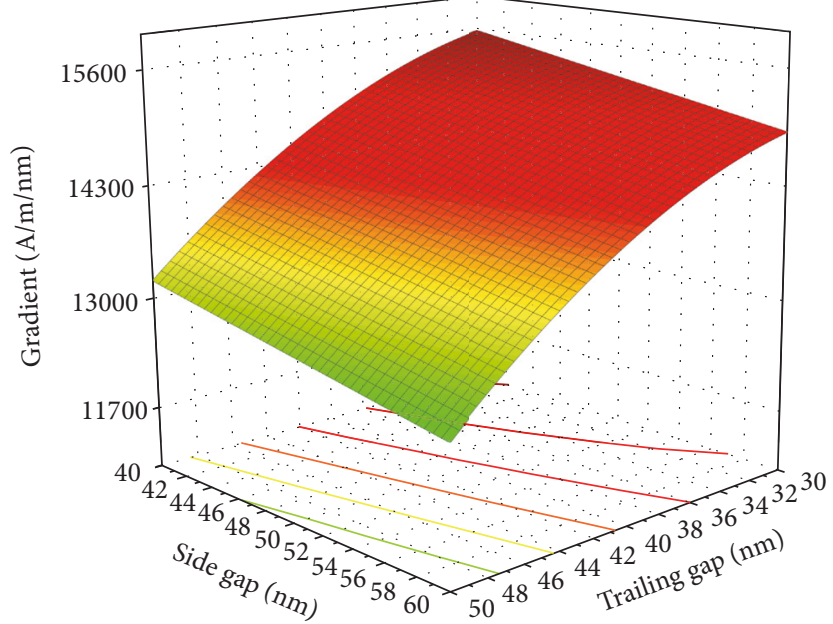

(a)

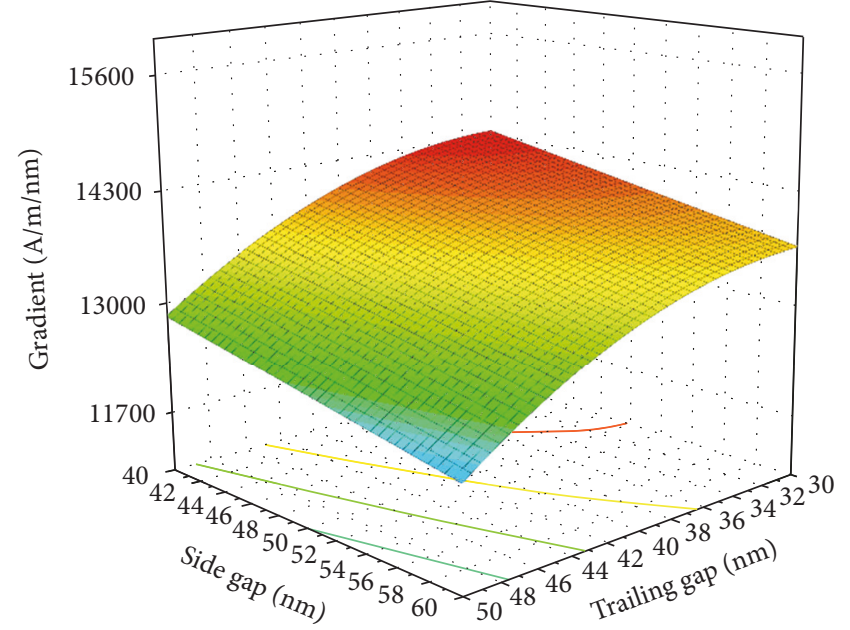

(b)

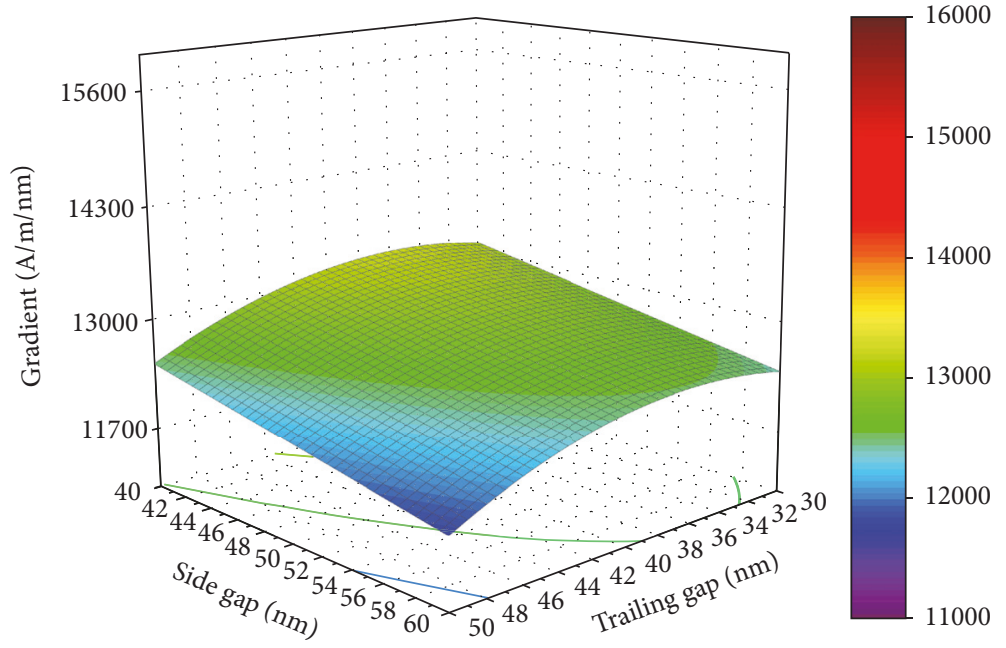

(c)

FIgURE 7: The response surface of the EWF gradient with respect to SG and TG with media $K_{u}=6 \times 10^{5} \mathrm{~J} / \mathrm{m}^{3}(\mathrm{a}), 5.5 \times 10^{5} \mathrm{~J} / \mathrm{m}^{3}(\mathrm{~b})$, and $5 \times$ $10^{5} \mathrm{~J} / \mathrm{m}^{3}$ (c).

the EWF gradient. Moreover, the gradient sensitivity to the shield's geometric design parameters is dependent on the coercivity of the medium. The proposed method is useful for evaluation of the bit error rate and SNR of shingled bitpatterned recording systems.

\section{Conflicts of Interest}

The authors declare that there are no conflicts of interest regarding the publication of this paper.

\section{References}

[1] K. Z. Gao, O. Heinonen, and Y. Chen, "Read and write processes, and head technology for perpendicular recording," Journal of Magnetism and Magnetic Materials, vol. 321, no. 6, pp. 495-507, 2009.
[2] M. H. Kryder, E. C. Gage, T. W. Mcdaniel et al., "Heat assisted magnetic recording," Proceedings of the IEEE, vol. 96, no. 11, pp. $1810-1835,2008$

[3] S. Okamoto, N. Kikuchi, M. Furuta, O. Kitakami, and T. Shimatsu, "Microwave assisted magnetic recording technologies and related physics," Journal of Physics D: Applied Physics, vol. 48, no. 35, Article ID 353001, 2015.

[4] M. Zhang, T. Zhou, and Z. Yuan, "Analysis of switchable spin torque oscillator for microwave assisted magnetic recording," Advances in Condensed Matter Physics, vol. 2015, Article ID 457456, 6 pages, 2015.

[5] R. Wood, M. Williams, A. Kavcic, and J. Miles, “The feasibility of magnetic recording at 10 terabits per square inch on conventional media," IEEE Transactions on Magnetics, vol. 45, no. 2, pp. 917-921, 2009.

[6] A. Aghayev, M. Shafaei, and P. Desnoyers, "Skylight - a window on shingled disk operation," ACM Transactions on Storage, vol. 11, no. 4, article 16, 2015. 
[7] A. Kikitsu, "Prospects for bit patterned media for high-density magnetic recording," Journal of Magnetism and Magnetic Materials, vol. 321, no. 6, pp. 526-530, 2009.

[8] T. R. Albrecht, H. Arora, V. Ayanoor-Vitikkate et al., "Bitpatterned magnetic recording: theory, media fabrication, and recording performance," IEEE Transactions on Magnetics, vol. 51, no. 5, 2015.

[9] S. Wang, Y. Wang, and R. H. Victora, "Shingled magnetic recording on bit patterned media at $10 \mathrm{~Tb} / \mathrm{in}^{2}$," IEEE Transactions on Magnetics, vol. 49, no. 7, pp. 3644-3647, 2013.

[10] A. Kovacs, H. Oezelt, M. E. Schabes, and T. Schrefl, "Numerical optimization of writer and media for bit patterned magnetic recording," Journal of Applied Physics, vol. 120, no. 1, Article ID 013902, 2016.

[11] C. Vogler, C. Abert, F. Bruckner, D. Suess, and D. Praetorius, "Heat-assisted magnetic recording of bit-patterned media beyond $10 \mathrm{~Tb} / \mathrm{in}^{2}$," Applied Physics Letters, vol. 108, no. 10, Article ID 102406, 2016.

[12] G. Yu and J. Chen, "Skew effect-induced track erasure of shingled magnetic recording system," IEEE Transactions on Magnetics, vol. 50, no. 11, pp. 1-4, 2014.

[13] R. H. Victora, S. M. Morgan, K. Momsen, E. Cho, and M. F. Erden, "Two-dimensional magnetic recording at 10 Tbits/in ${ }^{2}$, IEEE Transactions on Magnetics, vol. 48, no. 5, pp. 1697-1703, 2012.

[14] K. Keng Teo, M. R. Elidrissi, K. S. Chan, and Y. Kanai, "Analysis and design of shingled magnetic recording systems," Journal of Applied Physics, vol. 111, no. 7, Article ID 07B716, 2012.

[15] Y. Kanai, Y. Jinbo, T. Tsukamoto, S. J. Greaves, K. Yoshida, and H. Muraoka, "Finite-element and micromagnetic modeling of write heads for shingled recording," IEEE Transactions on Magnetics, vol. 46, no. 3, pp. 715-721, 2010.

[16] X. M. Yang, S. Xiao, Y. Hsu, M. Feldbaum, K. Lee, and D. Kuo, "Directed self-assembly of block copolymer for bit patterned media with areal density of 1.5 Teradot/Inch ${ }^{2}$ and beyond," Journal of Nanomaterials, vol. 2013, Article ID 615896, 17 pages, 2013.

[17] W. Tipcharoen, A. Kaewrawang, and A. Siritaratiwat, "Design and micromagnetic simulation of $\mathrm{Fe} / \mathrm{L1}_{0}-\mathrm{FePt} / \mathrm{Fe}$ trilayer for exchange coupled composite bit patterned media at ultrahigh areal density," Advances in Materials Science and Engineering, vol. 2015, Article ID 504628, 5 pages, 2015.

[18] H. Oezelt, A. Kovacs, P. Wohlhüter et al., "Micromagnetic simulation of exchange coupled ferri-/ferromagnetic composite in bit patterned media," Journal of Applied Physics, vol. 117, no. 17, Article ID 17E501, 2015.

[19] R. H. Myers and D. C. Montgomery, Response Surface Methodology: Process and Product Optimization Using Designed Experiments, John Wiley \& Sons Inc., Hoboken, NJ, USA, 2009.

[20] T. Kagami, "An areal-density capability study of SMR by using improved write and read heads," in Proceedings of the Intermag, FA-01, April 2011.

[21] M. Salo, T. Olson, R. Galbraith et al., "The structure of shingled magnetic recording tracks," IEEE Transactions on Magnetics, vol. 50, no. 3, pp. 18-23, 2014.

[22] K. Miura, E. Yamamoto, H. Aoi, and H. Muraoka, "Skew angle effect in shingled writing magnetic recording," Physics Procedia, vol. 16, pp. 2-7, 2011.

[23] E. C. Stoner and E. P. Wohlfarth, "A mechanism of magnetic hysteresis in heterogeneous alloys," Philosophical Transactions of the Royal Society A: Mathematical, Physical and Engineering Sciences, vol. 240, no. 826, pp. 599-642, 1948.
[24] H. Oezelt, A. Kovacs, J. Fischbacher et al., "Switching field distribution of exchange coupled ferri-/ferromagnetic composite bit patterned media," Journal of Applied Physics, vol. 120, no. 9, Article ID 093904, 2016.

[25] "Half-normal probability plot, NIST/SEMATECH e-handbook of statistical methods," 2012, http://www.itl.nist.gov/div898 /hand-book/. 

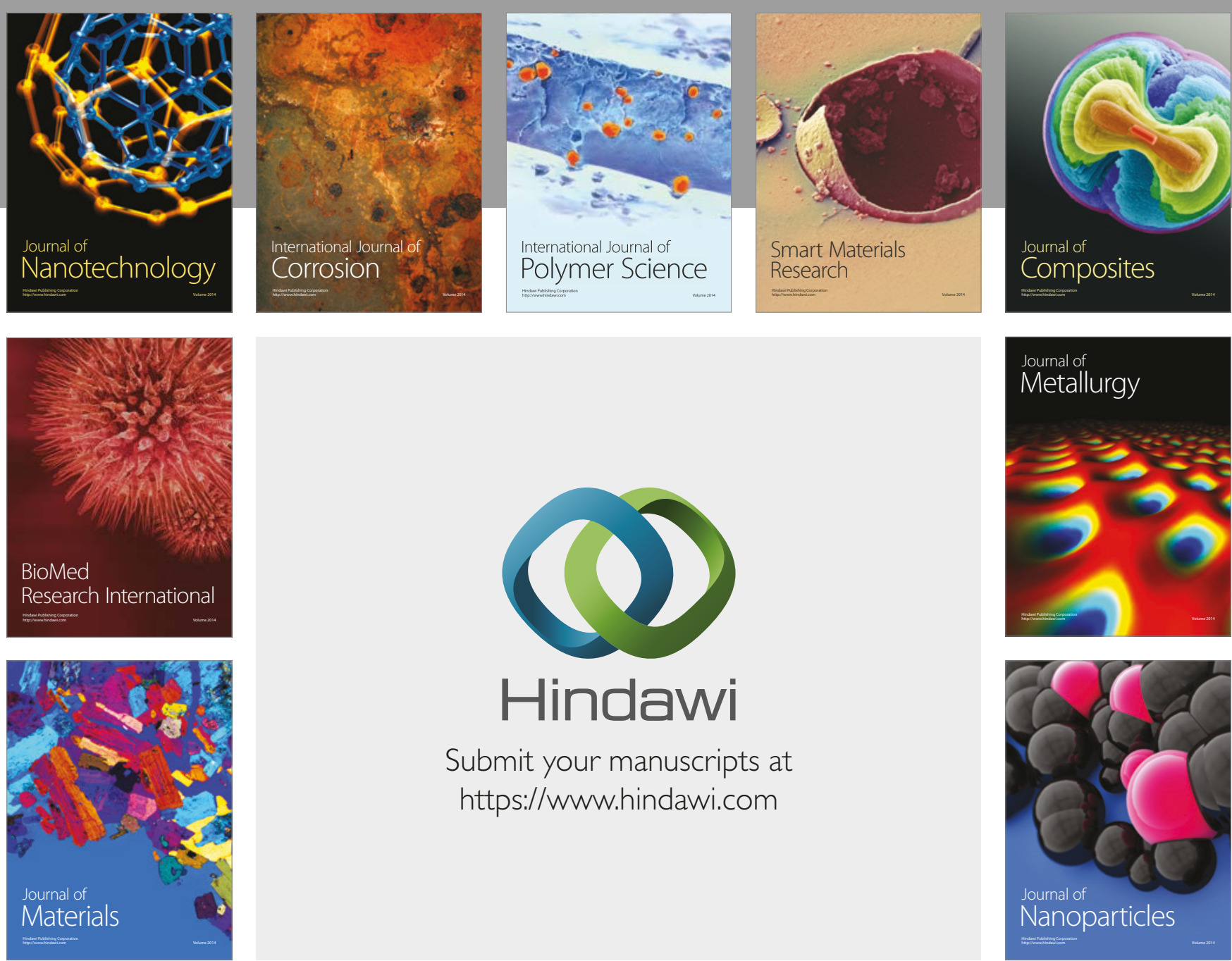

\section{Hindawi}

Submit your manuscripts at

https://www.hindawi.com
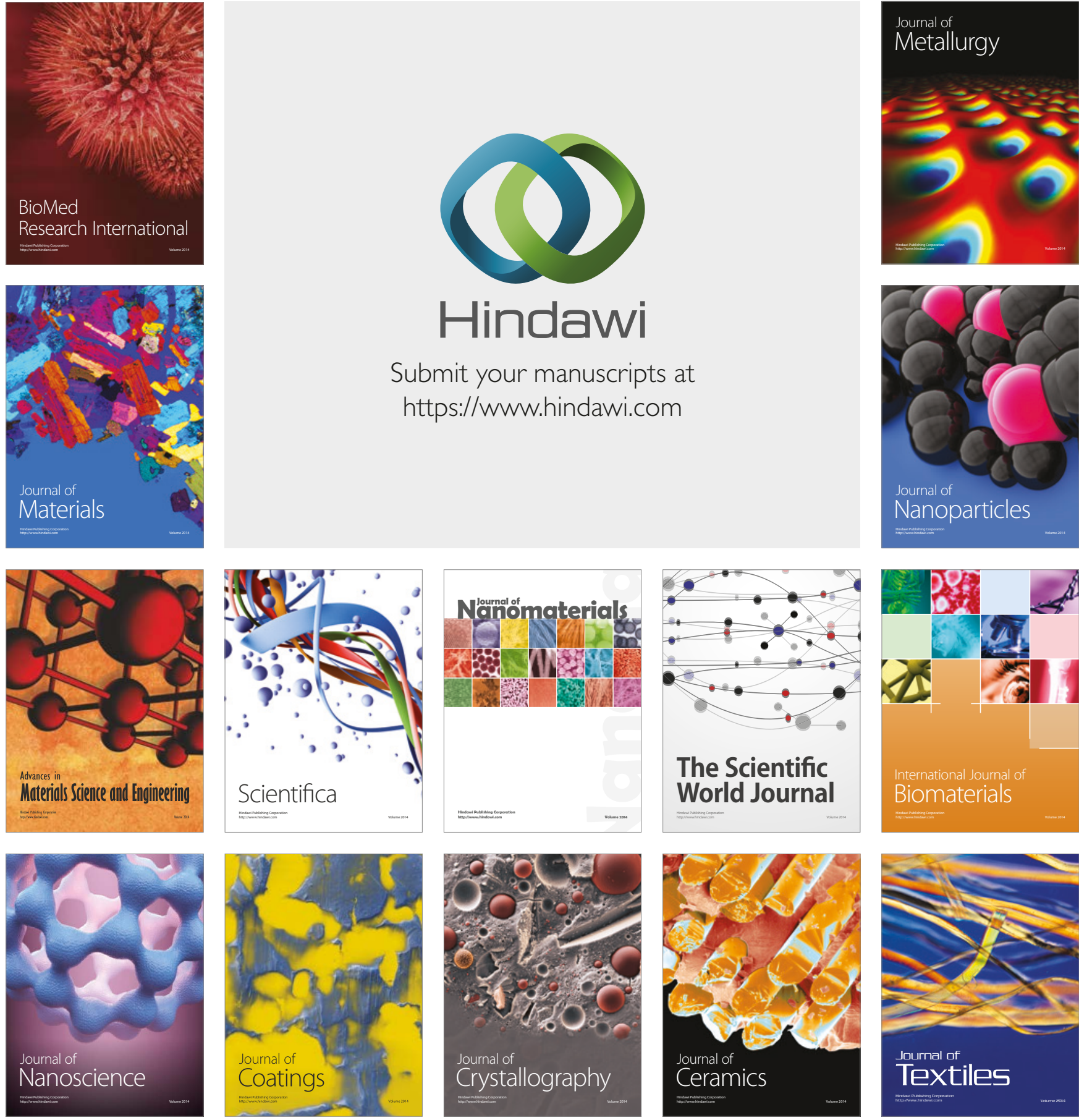

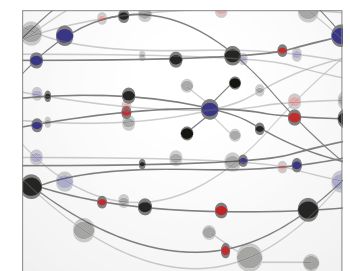

The Scientific World Journal
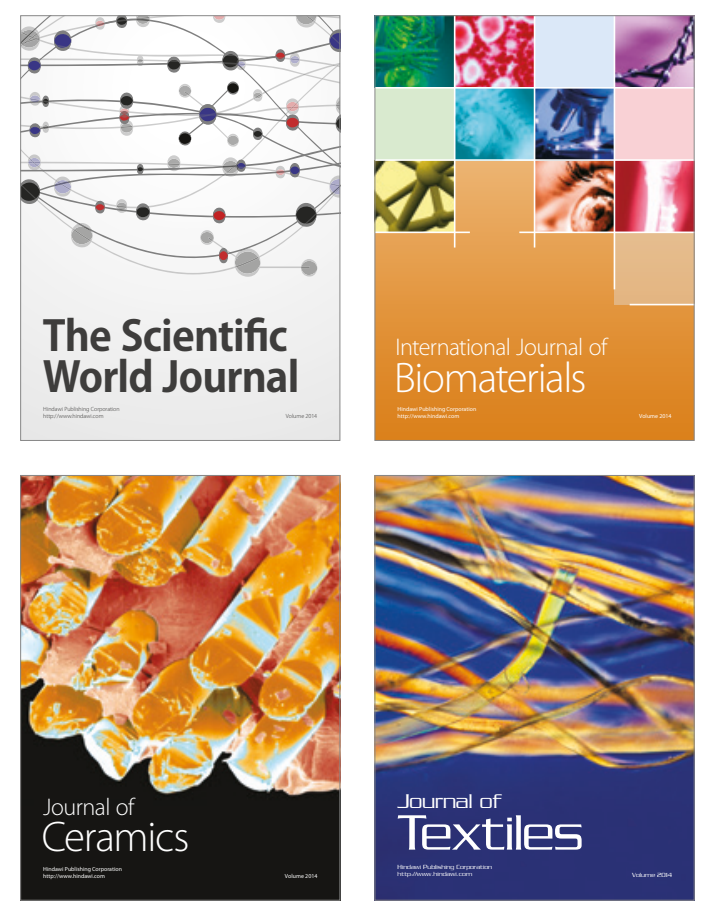